\title{
Risk Factors for Recurrence of Hepatocellular Carcinoma after Curative Resection
}

\author{
Mitsugi Shimoda, Tsunehiko Maruyama, Kazuomi Suzuki, Tomoya Tago, Kiyotaka Nishida, \\ Jiro Shimazaki, Shuji Suzuki
}

Corresponding author:

Mitsugi Shimoda, M.D., Ph.D.

3-20-1, Chuo, Ami, Ibaraki

300-0395, Japan

Tel: $\quad+81-29-887-1161$

Fax: $+81-29-840-2089$

E-mail: mshimoda@tokyo-med.ac.jp
Gastroenterological Surgery, Tokyo Medical University, Ibaraki Medical Center

\section{ABSTRACT}

Background/Aims: Hepatic surgical resection is one of the most effective treatment for hepatocellular carcinoma (HCC). However, recurrence of HCC (HCCR) is still very common among operated patients, and its risk factors still remains unclear.

Materials and Methods: We have conducted a retrospective study of 56 patients operated for HCC in our medical center during April 2008 through March 2016, to analyze the risk factors for HCCR. The ideal cutoff values of laboratory parameters was defined using the Receiver Operating Characteristic (ROC) curve analysis, and logistic regression model analyses were used to calculate odds ratios with $95 \% \mathrm{Cl}$. P values less than 0.05 were considered as statistically significant.

Results and Conclusion: All 56 patients accrued to this retrospective study underwent curative resection of HCC. The patients compromised of 11 female and 45 male, with the median age of 68.5years. Twenty-three out of 56 (40.1\%) patients experienced HCC recurrence, and the Median time of recurrence was 14.5 months. The 5-year cancer-free survival rate was $42.5 \%$. Univariate analysis showed that the use of intermittent Pringle Maneuver (IPM: $p=0.036$ ), limited resection (LR: $p=0.011$ ), and cancerous infiltration of the capsule (fc-inf; $p=0.017$ ), were associated with HCC recurrence. According to the multivariate analysis, using IPM $(p=0.008), \operatorname{LR}(p=0.012)$, and $f c-i n f(p=0.014)$, were risk factors for patients with HCCR.

Key words: hepatocellular carcinoma, risk factors, recurrence

\section{BACKGROUND/AIMS}

Hepatocellular carcinoma (HCC) is one of the most common form of cancer worldwide, and is the fourth leading cancer deaths in Japan (1). HCC related cancer death was steady decreasing, and its survival rate was improving in the last decade, as well as the survival rate of resection cases (54.2\%). Currently the best treatment for HCC is to perform radical treatment, and curative resection is the first-choice therapeutic option in Japan (2). Overall survival after resection of HCC has made extreme progress in the last decade, however, the recurrence 
rate exceeds $80 \%$ even after curative resection (3). The risk factors for postoperative recurrence of HCC (HCCR) are classified into 3 categories: (1) tumor factors such as tumor size and numbers (2) host factors such as the presence of cirrhosis and hepatitis viral load, and (3) surgical and pathological factors such as surgical margin and microvascular (portal vein or hepatic vein) invasions $(4,5)$. Some reports published recently describes of various inflammation-based prognostic scores, such as Glasgow prognostic score (GPS) and neutrophil to lymphocyte ratio (NLR), to have been associated with overall as well as cancer free survival in patients with several types of cancer including HCC (6-10).

In this retrospective study, we have evaluated and assessed the risk factors for HCCR usingperioperative and pathological factors.

\section{PATIENTS AND METHODS}

\section{Study population}

We have accrued patients who had single lesion and underwent initial curative resection of the liver at the Department of Gastroenterological Surgery, Ibaraki Medical center, Tokyo Medical University during April 2008 and March 2016. Patient records were collected and analyzed retrospectively. The study was conducted in accordance with the Declaration of Helsinki (1975, as revised in 2008) and the regulations of the Japanese Ministry of Health, Labour and Welfare. The ethics committee of Tokyo Medical University Ibaraki Medical Center approved this retrospective study protocol, and written informed consent was provided from all study participants.

Curative resection (RO resection) was defined as that leaving behind no gross or microscopic tumor on the cut surface and in the remnant liver. Clinical and pathologic staging was performed according to the General Rules for the Clinical and Pathological Study of Primary Liver Cancer (third edition).

There were 56 patients who have received initial liver resection at our department during the study period, among which 47 of 56 cases had single lesion $\mathrm{HCC}$. For this retrospective analysis, the patients were divided into two groups based on whether the patients experienced HCC recurrence (HCCR G: $n=23$ ) or no HCC recurrence (nHCCRG: $n=33$ ), and the patients' clinicopathologic and operative factors associated with HCCR were evaluated in all of the 56 patients. HCC was diagnosed before operation using radiological examinations multi-detector row computed tomography (MDCT), magnetic resonance imaging (MRI) and/or abdominal ultrasound (US). The decision for liver resection was determined based on each patients' liver functional reserve mainly assessed by using the functional hepatic resection rate, calculated using 3DCT/99 mTc-galactosyl-human serum albumin single-photon emission CT fusion imaging (11). We also incorporated Makuuchi Criteria, which comprises of preoperative measurements of ascites, serum bilirubin level, and indocyanine green retention rate at 15 min (ICG R15) after administration from 2014.

All HCC were diagnosed histolopathologically, and combined CCC were excluded in this study. The histologic grade of tumor differentiation was assigned according to the Edmondson grading system. Clinicopathologic factors such as age, gender, hepatitis, tumor markers (serum alpha-fetoprotein: AFP), liver cirrhosis, type of liver resection (anatomical resection: AR or limited resection: $L R$ ), neutrophil to lymphocyte ratio (NLR), Glasgow prognostic score (GPS), operative time, amount of blood loss, use of intermitted Pringle maneuver (IPM), tumor size, tumor differentiation, pathological stage, and pathological microvascular invasion, were incorporated.

\section{Operative procedure}

After confirmation of intrahepatic metastasis, we have performed US to verify the location of HCC via laparotomy.

For AR patients, the accurate resection area in accordance to Couinaud classification was defined through staining techniques and intraoperative ultrasound (12), and/or Glissonean pedicle approach for sectionectomy, hemihepatectomy, and subsegmentectomy. Issues concerning the tertiary branches that originates from the deep portions of the secondary pedicles, such as segment 7 or 8 , that cannot be approached from the hepatic hilus, were managed by initially dissecting the liver parenchyma on the border between the sections, and then by exposing and dividing each Grissonean pedicles $(13,14)$.

The liver was dissected along a line so to secure the surgical margin of at least $2 \mathrm{~cm}$ where possible for $L R$ patients. Final decision to perform LR in operating room was based on factors such as tumor location confirmed through ultrasound, and liver condition (with or without cirrhosis). When AR was impossible, we took into account full exposure of the landmark vessels on the cutting surface, such as the right and middle hepatic veins, and have divided the liver parenchyma by an ultrasonic dissector. We initially started liver transection under no Pringle maneuver, but when it was difficult to 
control bleeding during transection of parenchyma, we used the IPM ( 10 minutes clamp and 5 minutes release or 15 minutes clamp and 5 minutes release). Drainage tubes were removed when there was no visible bile leakage, and when the fluid bilirubin level was less than $2.0 \mathrm{mg} / \mathrm{dl}$.

\section{Follow up}

All patients were followed up in outpatient clinic from initial liver resection until either death or recurrence. We evaluated recurrence as follows: monthly monitoring using measurements of AFP and/or PIVKA-II, and every 3 months by MDCT or US. All of the patients were followed from 6 months up to 8 years after surgery. For those applicable patients who had HCCR, we selected trans-catheter arterial chemoembolization, or Sorafenib.

The GPS was estimated as follows; patients with both an increased CRP level (> $1.0 \mathrm{mg} / \mathrm{dL}$ ) and hypoalbuminemia $(<3.5 \mathrm{~g} / \mathrm{dL})$ were allocated a score of 2 , patients with only one of these biochemical abnormalities were allocated a score of 1 , and patients with neither of these abnormalities were allocated a score of 015. The NLR was defined as the absolute neutrophil count divided by the absolute lymphocyte count (6).

Each of the clinic-pathological characteristics of both groups were compared among both groups.

\section{Statistics}

Statistical analyses were performed with the SPSS statistical software package (version 24.0, SPSS Inc, Chicago, IL). Receiver operating characteristic (ROC) curve analysis was used to define the ideal cut off values of laboratory parameters (such as, NLR, AFP, amount of bleeding operating time and tumor size). The recommended cut off value for each parameter was determined as the most prominent points on the ROC curve for sensitivity and specificity, respectively. Cancer free Survival analysis was performed using Kaplan-Meier analysis. Univariate and multivariate analyses were performed to clarify the laboratory parameter most significantly associated with HCCR, and were used to assess risk factors to predict the HCCR after resection. Univariate analyses, Mann-Whitney U-test, and Fisher's exact test were utilized, and Odds ratios with $95 \% \mathrm{Cl}$ were calculated using logistic regression model analyses. P values of less than 0.05 were considered to be statistically significant.

\section{RESULTS AND CONCLUSION}

Eleven of 56 patients (19.6\%) were LR, and 45 patients were AR (80.4\%); 19 patients had subsegmentectomy, 19 had sectinectomy, and seven patients had hemihepatectomy.

One patient had died within 30 days after surger due to liver dysfunction. All of 56 patients received pathological margin negative resection during the study period. Twenty-three of 56 patients (41.1\%) were HCCR, 22 patients had intrahepatic alone recurrence; one patient had extrahepatic recurrence in lung.

\section{Cancer free patients survival (CFS)}

Median time of recurrence was 14.5 months (range: 2.0-85.1 months), and the 1-, 3- and 5-year CFS rates were $71.6,62.0$ and 42.5 per cent respectively (fig. 1).

\section{Univariate analysis}

\section{Preoperative factors}

There were no significant differences in all of factors between the two groups (table 1).

\section{Operative factors}

LR ( $p=0.011, O R=5.58,95 \% \mathrm{Cl}=1.47-21.12)$ and with IPM ( $p=0.036, O R=3.28,95 \% \mathrm{Cl}=1.08-9.99)$ were significant risk factors for HCCR (table 2).

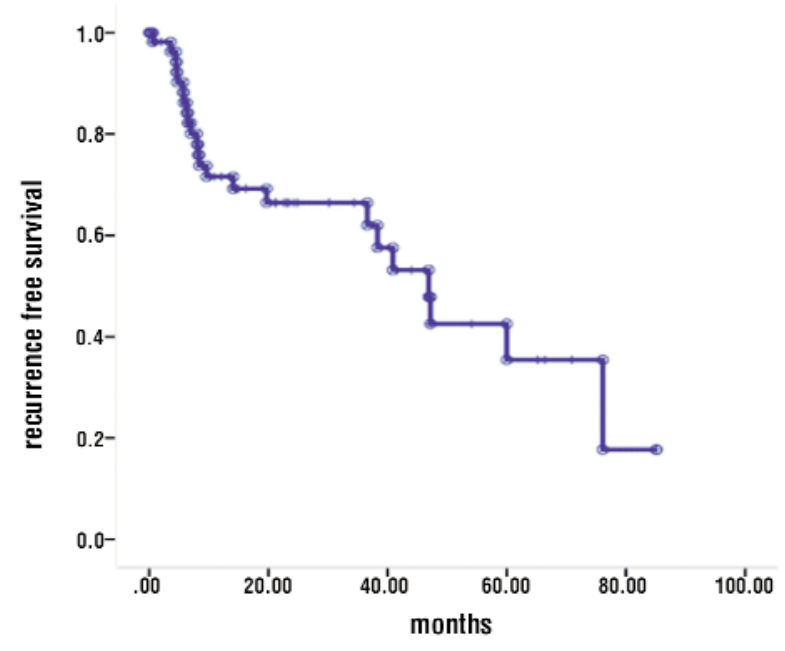

Figure 1 - Kaplan-Meier estimates of cancer free patient's survival in curative resection for HCC 
Table 1 - Univariate analysis for HCC Recurrence after curative resection from logistic regression model analyses in preoperative factors

\begin{tabular}{lcccc}
\hline & HCCR $(\mathbf{n}=23)$ & nHCCR $(\mathbf{n}=33)$ & Odd & 95\% C.I. p \\
\hline Gender $($ F/M) & $2 / 21$ & $9 / 24$ & 0.25 & $0.05-1.310 .101$ \\
\hdashline Age $(68.5$ yrs $\geq /<)$ & $20 / 3$ & $24 / 9$ & 0.40 & $0.10-1.680 .211$ \\
\hdashline Hepatitis $( \pm)$ & $19 / 4$ & $26 / 7$ & 0.78 & $0.20-3.060 .742$ \\
\hline HCV $( \pm)$ & $19 / 4$ & $26 / 7$ & 1.13 & $0.38-3.370 .833$ \\
\hdashline HBV $( \pm)$ & $6 / 17$ & $5 / 28$ & 0.57 & $0.13-1.920 .316$ \\
GPS $(0 / 1,2)$ & $14 / 9$ & $23 / 9$ & 1.64 & $0.53-5.130 .393$ \\
\hdashline NLR $(1.74 \geq /<)$ & $6 / 17$ & $16 / 17$ & 0.38 & $0.13-1.190 .096$ \\
Child-Pugh $(\mathrm{A} / \mathrm{B})$ & $20 / 3$ & $31 / 2$ & 0.43 & $0.07-2.810 .378$ \\
\hdashline AFP $(34 \mathrm{ng} / \mathrm{ml}: \geq /<)$ & $7 / 16$ & $10 / 23$ & 1.01 & $0.32-3.200 .992$ \\
\hline
\end{tabular}

Table 2 - Univariate analysis for HCC Recurrence after curative resection from logistic regression model analyses in operative factors

\begin{tabular}{lcccc}
\hline & HCCR $(\mathbf{n}=23)$ & nHCCR $(\mathbf{n}=33)$ & Odd & 95\% C.I. p \\
\hline Amount of bleeding $(589 \mathrm{ml}: \geq /<)$ & $9 / 14$ & $18 / 15$ & 1.87 & $0.63-5.510 .258$ \\
\hdashline Operation time $(435 \mathrm{~min}:>/<)$ & $3 / 20$ & $6 / 27$ & 1.41 & $0.31-6.340 .656$ \\
\hdashline Procedure $(\mathrm{LR} / \mathrm{AR})$ & $7 / 16$ & $4 / 29$ & 5.58 & $1.47-21.120 .011$ \\
\hline Pringle maneuver $( \pm)$ & $7 / 16$ & $16 / 17$ & 3.28 & $1.08-9.990 .036$ \\
\hline
\end{tabular}

LR: limited resection, AR: anatomical resection

\section{Histopathological factors}

Only cancerous infiltration of the capsule (fc-inf: $\mathrm{p}=0.017, \mathrm{OR}=4.00,95 \% \mathrm{Cl}=1.28-12.47)$ was significant risk factor for HCCR (table 3).

\section{Multivariate analysis}

Multivariate analysis using the 6 clinical and surgical characteristics selected above, revealed that $L R(p=0.012$, Odd ratio $=8.05,95 \% \mathrm{Cl}=1.58-40.93)$, with IPM ( $p=0.008$,

Table 3 - Univariate analysis for HCC Recurrence after curative resection from logistic regression model analyses in histopathological factors

\begin{tabular}{|c|c|c|c|c|}
\hline & HCCR $(n=23)$ & nHCCR $(n=33)$ & Odd & 95\% C.I. p \\
\hline Differentiation (mode./ other) & $18 / 5$ & $30 / 3$ & 2.78 & $0.59-13.040 .195$ \\
\hline Tumor size $(2.7 \mathrm{~cm}: \geq /<)$ & $12 / 11$ & $16 / 17$ & 1.16 & $0.40-3.360 .786$ \\
\hline Tumor number $(1 / \geq 2)$ & $19 / 4$ & $28 / 5$ & 1.18 & $0.28-4.970 .822$ \\
\hline fc $( \pm)$ & $21 / 2$ & $23 / 10$ & 4.57 & $0.90-23.290 .068$ \\
\hline fc inf $( \pm)$ & $16 / 7$ & $12 / 21$ & 4.00 & $1.28-12.470 .017$ \\
\hline$S C( \pm)$ & $19 / 4$ & $28 / 5$ & 1.18 & $0.28-4.970 .822$ \\
\hline$S( \pm)$ & $20 / 3$ & $29 / 4$ & 1.09 & $0.22-5.400 .918$ \\
\hline $\mathrm{vp}( \pm)$ & $7 / 16$ & $5 / 28$ & 2.45 & $0.67-9.010 .177$ \\
\hline $\mathrm{VV}( \pm)$ & $5 / 18$ & $3 / 30$ & 2.78 & $0.59-13.10 .195$ \\
\hline$b( \pm)$ & $2 / 21$ & $2 / 31$ & 1.48 & $0.19-11.320 .708$ \\
\hline $\operatorname{im}( \pm)$ & $4 / 19$ & $1 / 32$ & 6.74 & $0.70-64.800 .099$ \\
\hline $\operatorname{sm}( \pm)$ & $1 / 22$ & $7 / 26$ & 5.92 & $0.68-51.920 .108$ \\
\hline Stage (I,II,III / IV) & $18 / 5$ & $32 / 1$ & 8.88 & $0.96-82.120 .054$ \\
\hline Liver cirrhosis $( \pm)$ & $18 / 5$ & $22 / 11$ & 1.80 & $0.53-6.140 .348$ \\
\hline
\end{tabular}

fc: capsule formation, fc-inf: cancerous infiltration of the capsule, s: invasion of the serosa, vp: portal vein invasion, v: hepatic vein invasion, b: bile duct invasion, im: intrahepatic metastasis, sm: surgical margins 
Table 4 - Multivariate analysis for HCC Recurrence after curative resection from logistic regression model analyses operative and histopathological factors

\begin{tabular}{lccc}
\hline & Odd & 95\% C.I. & p \\
\hline Procedure $(\mathrm{LR} / \mathrm{AR})$ & 8.05 & $1.58-40.93$ & 0.012 \\
\hline Pringle maneuver $( \pm)$ & 7.31 & $1.66-32.06$ & 0.008 \\
\hdashline fc inf $( \pm)$ & 5.79 & $1.43-23.45$ & 0.014 \\
\hline
\end{tabular}

fc-inf: cancerous infiltration of the capsule, LR: limited resection, AR: anatomical resection

Odd ratio $=7.31,95 \% \mathrm{Cl}=1.66-32.06)$, and fc-inf ( $\mathrm{p}=0.014$, Odd ratio=5.79, 95\% $\mathrm{Cl}=1.43-23.45)$ were significant prognostic factors for HCCR (table 4).

Surgical resection is a curative treatment modality for HCC. However, the major issue after surgical resection is the high rate of HCCR; the risk of tumor recurrence following curative resection is $70 \%$. Therefore, preventing HCCR is still critical in improving the survival of patients who undergo liver resection. This makes it important to select patients appropriately and to analyze factors related to prognosis in order to improve the recurrence free rates. In this study, we have evaluated and identified the risk factors for HCCR after curative resection to be surgical procedure of limited resection, usage of IPM, and whether or not the pathological cancer cells were infiltrating into the capsule.

In the last decade, several reports already have described and identified numerous independent factors for HCCR. Postoperative HCCR is thought to take place in two ways: intrahepatic metastasis in the residual liver, and metachronous, multicentric hepatocarcinogenesis based on hepatitis (16). High level of preoperative AFP, macrovascular and microvascular invasion, surgical procedures, Edmondson-Steiner grade III or IV and some gene expressions, were identified as a predictor or risk factors for $\operatorname{HCCR}(17,18)$. Unfortunately, such as above well-known predictors were not independent risk factors for HCCR in our study. Only three factors, such as Pringle maneuver, limited resection and cancerous infiltration of the capsule became significant risk factors for HCCR.

Surgical procedure, especially $A R$, was one of the most independent factors for the prevention of HCCR $(19,20)$. AR was originally introduced as sectionectomy or subsegmentectomy, by Makuuchi et al (12) of a hepatic segment defined by tumor-bearing portal branches. Because of the high likelihood of cancer cells from HCC spreading through the portal venous system, it was effective for eradication of intrahepatic metastases from HCC. On the other hand, some reports suggested that there is no superiority of $A R$ to $L R$ relevant to the CFS in patients with a single HCC (21, 22). For the moment, clear decision cannot be made on whether AR or LR better contributes to the prevention of $\operatorname{HCCR}(23,24)$. We recommend that a randomized control study be conducted as the next step.

The Pringle maneuver, a portal pedicle clamping technique, is a classical surgical technique that has been used during liver surgery in patients with HCC (25). This technique significantly prevents the risk of intra-operative bleeding in comparison with none portal clamping maneuver. On the other hand, it is associated with some disadvantage factors for the patients, such as ischemia reperfusion injury, prolonged operation time, and excessive surgery related complications. Furthermore, experimental models have shown that Pringle maneuver to the liver promotes cancer cell growth, and may cause delayed damage to the residual liver, there by accelerating tumor recurrence (26). Recently some clinical reports that followed this experimental hypothesis argue that a prolong Pringle maneuver time, or in fact the application of Pringle maneuver,may become one of the risk factor for recurrence of $\operatorname{HCC}(27,28)$. In present study, we have identified that IPM was an independent risk factor adversely affecting the CFS of patients with HCC. The IPM induced an ischemia reperfusion injury detrimental to the outcome of patients undergoing liver resection for HCC. On the other hand, amount of blood loss is one of the important risk factor for CFS in patients with HCC. From our results, we recommend that IPM should be avoided in cases with limited resection.

Some of pathological factors are often taken as an important risk factor for the $\operatorname{HCCR}(29,30)$. In our series, cancerous infiltration of the capsule was also accounted as an independent risk factor for HCCR. As the pathological factors including micro vascular invasion cannot be evaluated before surgery, these factors will be used as an indicator to consider adjuvant treatment after $\mathrm{HCC}$ resection.

This study had several limitations. It was a retro- 
spective and a single center study therefore the number of patients accruable to the study was limited. A prospective multicenter study with larger patient population is necessary to confirm and verify our results.

In our result, LR, using IPM and positive fc-inf became risk factors for HCC recurrence. We would be to avoid IPM as an effective means to prevent recurrence.

\section{Conflict of interest}

\author{
All author declare that they have no conflict of \\ interest.
}

\section{REFERENCES}

1. Kudo M, Izumi N, Kokudo N, Matsui O, Sakamoto M, Nakashima O, et al. Management of hepatocellular carcinoma in Japan: Consensus-Based Clinical Practice Guidelines proposed by the Japan Society of Hepatology (JSH) 2010 updated version. Dig Dis. 2011;29(3):339-64.

2. Bruix J, Sherman M; Practice Guidelines Committee, American Association for the Study of Liver Diseases.Management of hepatocellular carcinoma. Hepatology. 2005;42(5):1208-36.

3. Navadgi S, Chang CC, Bartlett A, McCall J, Pandanaboyana S. Systematic review and meta-analysis of outcomes after liver resection in patients with hepatocellular carcinoma (HCC) with and without bile duct thrombus. HPB (Oxford). 2016;18(4):312-6.

4. Shirabe K, Kanematsu T, Matsumata T, Adachi E, Akazawa K, Sugimachi K. Factors linked to early recurrence of small hepatocellular carcinoma after hepatectomy: univariate and multivariate analyses. Hepatology. 1991;14(5):802-5.

5. Poon RT, Fan ST, Lo CM, Liu CL, Wong J. Long-term survival and pattern of recurrence after resection of small hepatocellular carcinoma in patients with preserved liver function: implications for a strategy of salvage transplantation. Ann Surg. 2002;235(3):373-82.

6. Walsh SR, Cook EJ, Goulder F, Justin TA, Keeling NJ. Neutrophillymphocyte ratio as a prognostic factor in colorectal cancer. J Surg Oncol. 2005;91(3):181-4

7. Gomez D, Farid S, Malik HZ, Young AL, Toogood GJ, Lodge JP, et al. Preoperative neutrophil-to-lymphocyte ratio as a prognostic predictor after curative resection for hepatocellular carcinoma. World J Surg. 2008;32(8):1757-62.

8. Ishizuka M, Oyama Y, Abe A, Kubota K. Combination of platelet count and neutrophil to lymphocyte ratio is a useful predictor of postoperative survival in patients undergoing surgery for gastric cancer. J Surg Oncol. 2014;110(8):935-41.

9. Feng JF, Huang Y, Chen QX. The combination of platelet count and neutrophil lymphocyte ratio is a predictive factor in patients with esophageal squamous cell carcinoma. Transl Oncol 2014;7(5):632-7.

10. McMillan DC. The systemic inflammation-based Glasgow Prognostic Score: a decade of experience in patients with cancer. Cancer Treat Rev 2013; 39(5):534-40.

11. Okabe H, Beppu T, Hayashi H, Mima K, Nakagawa S, Kuroki H, et al Rank classification based on the combination of indocyanine green retention rate at $15 \mathrm{~min}$ and (99m)Tc-DTPA-galactosyl human serum albumin scintigraphy predicts the safety of hepatic resection. Nucl Med Commun. 2014;35(5):478-83.

12. Makuuchi M, Hasegawa $\mathrm{H}$, Yamazaki $\mathrm{S}$. Ultrasonically guided subsegmentectomy. Surg Gynecol Obstet. 1985;161(4):346-50.

13. Yamamoto M, Katagiri S, Ariizumi S, Kotera Y, Takahashi Y, Egawa
H. Tips for anatomical hepatectomy for hepatocellular carcinoma by the Glissonean pedicle approach (with videos). J Hepatobiliary Pancreat Sci. 2014;21(8):E53-6.

14. Takasaki K. Glissonean pedicle transection method for hepatic resection: a new concept of liver segmentation. J Hepatobiliary Pancreat Surg. 1998;5(3):286-91.

15. Ishizuka M, Kubota K, Kita J, Shimoda M, Kato M, Sawada T. Impact of an inflammation-based prognostic system on patients undergoing surgery for hepatocellular carcinoma: a retrospective study of 398 Japanese patients. Am J Surg. 2012;203(1):101-6.

16. Poon RT, Fan ST, Ng IO, Lo CM, Liu CL, Wong J. Different risk factors and prognosis for early and late intrahepatic recurrence after resection of hepatocellular carcinoma. Cancer. 2000;89(3):500-7.

17. Zhu WJ, Huang CY, Li C, Peng W, Wen TF, Yan LN, et al. Risk factors for early recurrence of HBV-related hepatocellular carcinoma meeting milan criteria after curative resection. Asian Pac J Cancer Prev. 2013;14(12):7101-6.

18. Zheng H, Yang Y, Wang MC, Zheng H, Yang Y, Wang MC, et al. Low CDX1 expression predicts a poor prognosis for hepatocellular carcinoma patients after hepatectomy. Surg Oncol. 2016; 25(3):171-7.

19. Kim S, Kim S, Song I, Chun K. Comparison of survival outcomes after anatomical resection and non-anatomical resection in patients with hepatocellular carcinoma. Korean J Hepatobiliary Pancreat Surg. 2015;19(4):161-6.

20. Shimoda M, Tago K, Kato M, Aoki T, Kubota K. Prognostic Factors after Curative Resection for Single-Lesion Hepatocellular Carcinoma in Patients with Normal Liver Function: A Historical Cohort Study. Dig Surg. 2017;34(4):281-288.

21. Okamura Y, Ito T, Sugiura T, Mori K, Uesaka K. Anatomic versus nonanatomic hepatectomy for a solitary hepatocellular carcinoma: a case-controlled study with propensity score matching. J Gastrointest Surg. 2014;18(11):1994-2002.

22. Marubashi S, Gotoh K, Akita H, Takahashi H, Ito Y, Yano M, et al. Anatomical versus non-anatomical resection for hepatocellular carcinoma. Br J Surg. 2015;102(7):776-84.

23. Shimoda M, Tago K, Shiraki T, Mori S, Kato M, Aoki T, et al. Risk Factors for Early Recurrence of Single Lesion Hepatocellular Carcinoma After Curative Resection. World J Surg. 2016;40(10): 2466-71.

24. Yamamoto M, Takasaki K, Ohtsubo T, Katsuragawa H, Fukuda C, Katagiri S. Effectiveness of systematized hepatectomy with Glisson's pedicle transection at the hepatic hilus for small nodular hepatocellular carcinoma: retrospective analysis. Surgery. 2001;130(3):443-8.

25. Belghiti J, Noun R, Malafosse R, Jagot P, Sauvanet A, Pierangeli F, et al. Continuous versus intermittent portal triad clamping for liver resection: a controlled study. Ann Surg. 1999;229(3):369-75.

26. Man K, Lo CM, Xiao JW, Ng KT, Sun BS, Ng IO, et al. The significance of acute phase small-for-size graft injury on tumor growth and invasiveness after liver transplantation. Ann Surg. 2008;247(6): 1049-57.

27. Liu S, Li X, Li H, Guo L, Zhang B, Gong Z, et al. Longer duration of the Pringle maneuver is associated with hepatocellular carcinoma recurrence following curative resection. J Surg Oncol. 2016; 114(1):112-8.

28. Wang CC, lyer SG, Low JK, Lin CY, Wang SH, Lu SN, et al. Perioperative factors affecting long-term outcomes of 473 consecutive patients undergoing hepatectomy for hepatocellular carcinoma. Ann Surg Oncol. 2009;16(7):1832-42.

29. Ali MA, Li WF, Wang JH, Lin CC, Chen YJ, Lin TL, et al. Impact of pathological features of primary hepatocellular carcinoma on the outcomes of intrahepatic recurrence management: single center experience from Southern Taiwan. HPB (Oxford). 2016;18(10): 851-860.

30. Liu W, Wang K, Bao Q, Sun Y, Xing BC. Hepatic resection provided long-term survival for patients with intermediate and advancedstage resectable hepatocellular carcinoma. World J Surg Oncol. 2016;14:62. 